\title{
ON THE JOINT PRODUCTION OF RESEARCH AND TRAINING*
}

\section{Antonio FREITAS ${ }^{\dagger}$ and Inés MACHO-STADLER ${ }^{\ddagger}$}

May 20, 2014

\begin{abstract}
Universities and research institutions have the responsibility to produce science and to provide training to new generations of researchers. In this paper, we propose a model to analyze the determinants of a senior scientist's decisions about allocating time between these tasks. The results of this decision depend upon the characteristics of the research project, the senior scientist's concern for training and the expected innate ability of the junior scientist involved. We analyze the role that a regulator can play in defining both the value of scientific projects and the future population of independent scientists.
\end{abstract}

JEL codes: I23, D80

Keywords: Research and training, time allocation, junior scientist's mentoring

\section{Introduction}

It is widely accepted that universities and research institutions have the responsibility to produce and support science. One of the main missions of universities in the advancement

${ }^{*}$ We thank David Pérez Castrillo and a referee for their comments. We gratefully acknowledge the financial support from the research grants (ECO2009-07616 and ECO2012-31962) and Generalitat de Catalunya (2009SGR-169). The second author is fellow of MOVE.

${ }^{\dagger}$ Universitat Autònoma de Barcelona and NERA.

‡Universitat Autònoma de Barcelona and Barcelona GSE. 
of knowledge is the training the new generations of researchers. Their competence in performing this duty is crucial to assure high-quality research workforce in the future.

Considering that the training of junior researchers needs to be performed by people that have the capabilities to do research, in this paper we consider the incentives and dedication of senior scientists in providing training to junior scientists in a system of apprenticeship when the seniors are also involved in doing research (basic or applied). In particular, our paper is motivated by the increase in incentives to do research ${ }^{1}$ and the voices pointing out that research institutions may be failing in the training dimension, in the sense that there is a shortage of time (or the quality of the time) devoted to this task. To consider the reasons for this eventual shortage, we propose a model that focuses on the scientists allocation of time between research and training the next generation of researchers and we discuss the problems that may arise.

In many countries, there has been a considerable growth in the number of young people that go on to university education. ${ }^{2}$ In addition, the most prominent individuals in attaining an independent research status are in general $\mathrm{PhDs}$ and postdoctoral researchers who, thrive through more experience and skills in science, either in academics or in industry (Cech and Bond, 2004). ${ }^{3}$ Also, the literature on higher education (as the one on human resource management and mentoring) extensively recognizes the effects of training by senior staff in the competence, productivity, career development and independent skills of young professionals, in academia but also in industry.

The student-supervisor relationship is the most critical issue affecting the quality of the $\mathrm{PhD}$ training (which affects both success in obtaining the PhD degree and eventual job placement). In this process, the supervisors' guidance in early days of a PhD student, their

\footnotetext{
${ }^{1}$ In the US, the Bay-Dole Act puts incentices on research and transfer and commercialization of university innovations. In the UK, the Research Assesment Exercise (RAE) has an importnat impact on funding. Many countries university founding depends to certain extent on research results (publications, patents, licenses)

${ }^{2}$ For example, the "Patterns and Trends Report 2011 in UK Higher Education" reports that in the UK over the last 10 years (since 2000/01) the (full-time) postgraduate numbers have increased by 73.1 per cent (compared with an increase of 28.5 per cent for full-time undergraduates over the same period).

${ }^{3}$ Obviously, an alternative to training one's own researchers is to attract researchers trained elsewere. While this is an interesting idea, we choose to ignore this topic in this paper.
} 
knowledge about the area they are working in, and their involvement with their work play a key role (Pole et al., 1997). On a postdoctoral level, Vogel (1999) reports the experience of a principal investigator (PI) supervising postdocs in an internationally appraised lab. She states that the PI's key to producing successful and high-quality junior scientists is to provide them with original ideas and orientation, to encourage strong participation in the projects, and to listen to them to assess their skills, motivations and ambitions.

However, there are important concerns over issues such as thesis quality and completion rates. For these concerns supervision is an important part of the issue. The process of supervising junior research is not always smooth, and supervisors on occasion encounter difficulties to guide students, or less motivating to form students than the alternative use of their time. Student doctoral attrition remains a common problem in $\mathrm{PhD}$ training, and this is estimated at approximately $50 \%$ on the U.S. (Lovitts, 2001). In a case study about former students who spent at least two years in a PhD program, Golde (2000) identifies a lack of support and guidance from supervisors as one of the causes of attrition. The author is also able to identify characteristics for good supervision: the amount of time spent, the quality of interactions between student and supervisor, and an interest in the student's work. Accessibility seems to be an important issue as well. ${ }^{4}$ Training problems also occur at the postdoctoral level. Puljak (2006) reports that the most common complaint in postdoctoral training is ironically, the lack of postdoctoral training. Postdocs join a research lab and, shortly thereafter, many realize that they are on their own. It has also been identified that some advisors tend to take over the design of experiments, making postdocs feel like they are overeducated technicians. ${ }^{5}$

We study this issue by building a multitask model that examines the incentives of a senior scientist to train a junior scientist. In our framework, the senior scientist chooses

\footnotetext{
${ }^{4}$ It seems that there can be a mismatch in the perceptions of the supervisor and of doctoral students with respect to accessibility. In a study on the provisions of $\mathrm{PhD}$ training in biomedical research $\mathrm{PhD}$ programs, virtually all supervisors reported meeting frequently with their students, whereas $1 / 4$ of the students reported problems in accessing their supervisor (Frame and Allen, 2002).

${ }^{5}$ Nerad and Cerny (1999) also survey the perspective on postdoctoral employment in the U.S. and report that there exists a generalized discontent on behalf of postdoctoral researchers. The length of postdoctoral appointments has increased and these appointments are increasingly being seen as 'holding base', rather than being an important step in a young researcher's career.
} 
the time she will allocate to her own research and the time she will devote to train the junior scientist. We then evaluate the impact of time allocation on research quality and on the final skills of the junior scientist. For simplicity, the junior scientist is not an active player in our model (he does not make any decisions), but has a productive role in the project (he contributes to its final value). We assume that the junior scientist's final capabilities are affected by the training received from the senior scientist and also by his innate ability. To this respect, we abstract from information issues: senior and junior scientists have the same information about the junior scientist innate ability. ${ }^{6}$

Not surprisingly, when we analyze the senior's allocation of time between research and training, we find that when she has more time available she tends to devote more time to both tasks. Also, we show that when there is an increase in the innate ability of the junior scientist, an increase in the importance of training in the senior scientist's utility function, or a decrease in the productivity of the senior scientist in the project then there is a tendency to increase the time allocated to training. We also show that ex-ante ignorance about the true innate ability of the junior scientist may lead to more training for less able junior scientists, while there is a tendency toward an under-investment in training for the most talented ones.

As a robustness check, we analyze two extensions. First, we consider the case where the senior scientist can also spend time in selecting the junior scientist. Second, we examine the case where the senior scientist chooses the total amount of time she will work (and allocate to research and training), that is, the total amount of time spent on both tasks. We also discuss possible policy instruments for a regulator who is concerned about the value of projects and attaining highly qualified scientists in a desired proportion.

Several papers have considered the incentives for scientists to perform different tasks. For example, Lacetera and Zirulia (2008), in a context of corporate science with a great deal of competition, propose a model to explain the optimal choice of an effort to do

\footnotetext{
${ }^{6}$ This does not mean that the junior scientist's innate ability is public information. It is ex-ante unknown by both participants. Even though a student is selected to participate in a graduate programme or in a lab according to a GRE score, and other internal admission criteria of a department, significant uncertainty remains in predicting if a student has the potential to become a successful independent researcher (Lovitts, 2005).
} 
applied research and an effort to do basic research. They analyze the strength of incentives in the effort allocation decision of the scientist and the effects of different levels of competition. In Banal-Estañol and Macho-Stadler (2010)'s work, the authors present a model of incentives of a researcher who can choose to either allocate time between undertaking a new research idea or developing an existing one that will deliver immediate commercial benefits. In the same branch of the literature, Walckiers (2008) argues about whether it is more attractive for a university to produce both research and teaching. The author conducts his analysis in a contractual setting between the university (principal) and the academic/scientist (agent) and studies the incentives for university scientists to perform either one of the tasks or both of them. In contrast to Walckiers (2008), where the agent does teaching at the undergraduate level, we consider training at the graduate level which implies that there are complementarities among the two tasks. Walckiers (2008) uses an adverse selection framework, where researchers differ on their preference for both $\operatorname{tasks}^{7}$ and he shows that it can be optimal to produce research and teaching in the same institution (bundling the two tasks).

This paper is organized as follows. Section 2 describes the model and analyzes the equilibrium allocation of time to the two tasks: research and training. It also provides the comparative statics of the equilibrium efforts with respect to the parameters of the model. In Section 3, we evaluate and draw the patterns that the project's expected value and the junior scientist's final capability follow. We also present the ex-post ability of the junior scientist and the role of imperfect information in the distortions with respect to the full information and efficient outcomes. In Section 4 we perform a robustness check by considering two possibilities. First, we consider that the senior scientist can choose the total amount of time to exert in both tasks. Second, we consider the incentives for a senior scientist to spend time in previous activities that allow her to know more about the innate ability of the junior scientist. In Section 5 we discuss some policy instruments that may change the time allocation. In Section 6 we conclude. All proofs are in the Appendix.

\footnotetext{
${ }^{7}$ In our model, we could also discuss the researcher preferences, but this is not the main aspect of the analysis.
} 


\section{Basic Model}

We consider a senior scientist who is in charge of a research project and allocates her time (or her attention) between research and the training of a junior scientist under her supervision. We denote the research time or effort by $e_{R}$ and the training (guidance or education) effort by $e_{G}$. We assume that the senior scientist has limited time $t, t>0$, to allocate to these tasks. ${ }^{8}$ Formally, the senior scientist's time constraint is written as:

$$
e_{R}+e_{G}=t
$$

In our model, a junior scientist does not make any decisions (or equivalently, has not incentive problem and always exerts the highest effort)). A junior is endowed with an innate ability $\hat{a}$ and we assume that there is a population of junior scientists with different innate abilities. The innate ability of the junior takes values in the interval $[\underline{a}, \bar{a}]$, with $\bar{a}>\underline{a}>0$, and the expected innate ability is $E(\tilde{a})$. We will consider the case of perfect information where ex-ante the junior's ability is public information as a benchmark, and the case of ignorance where ex-ante the junior's ability is unknown by all the players since we think this is close to reality. In this last scenario, both the junior and the senior are uncertain about the true ability of the junior to do research. ${ }^{9}$

The senior scientist's vector of efforts affects two outcomes: the quality (the value) of the research project and the ex-post capability of the junior scientist she is training. The junior's final capability, denoted by $q$, is a function of his true innate ability $\hat{a} \in[\underline{a}, \bar{a}]$ and the training he receives $e_{G}$, and it is defined as follows:

$$
q=\hat{a} e_{G}
$$

We take the view that education provided by the senior is a necessary input to develop the junior's scientific capability: without training, even the most gifted junior scientist will not be able to acquire the capability to work on the research project in a profitable way (and maybe run a research project in the future).

\footnotetext{
${ }^{8}$ In Section 4, we endogenize the decision of the senior scientist concerning the time she works. For now, we assume that $t$ is exogenously given.

${ }^{9}$ In our model, there is always symmetric information about the junior scientist's innate ability. Under complete information senior and junior know that his innate ability is $\hat{a}$; under ignorance they expect it to be $E(\tilde{a})$.
} 
The project's value depends on the direct research effort exerted by the senior scientist and on the junior scientist's final capabilities. The scientific value of the senior's project is given by:

$$
v=(\alpha+\delta q) e_{R}
$$

where $\alpha, \alpha>0$, is the productivity of the senior's research time $e_{R}$, and $\delta, \delta \geq 0$, captures the synergies of working together with a junior scientist of capability $q$. In this sense, senior and junior scientists provide complementary inputs to the research project. Researchers may have different projects defined by $(\alpha, \delta)$ depending on their field and preferences, and we will discuss this further on.

The level $v$ may represent the publications obtained, patents achieved or other results of the discoveries such as licensing revenues and the success of a spin-off or a commercialization strategy. Note that by following this functional form, no value will be produced from the project if the senior scientist does not provide any research effort.

We assume that the senior scientist's utility function combines the project's value and the junior's final capability. Formally,

$$
u(v, \beta, q)=v+\beta q
$$

The project's value is included in the senior's preferences because it is a verifiable outcome that determines the senior scientist's payoff (in terms of tenure, promotion, research funds or salary). It is also a proxy for peer recognition and the "puzzle joy" (Stephan and Levin, 1992). The junior scientist's final capability enters the senior's utility in a proportion $\beta$, which represents the relative appreciation of the training outcome. We may also interpret that the second term is the senior's concern with the reputation attained from having a network and disciples who excel in the profession, where $\beta$ is a discount factor that weighs the effects of good training on future reputation improvement. ${ }^{10}$ For simplicity purposes, we assume $\beta \in[0, \delta t]$. The intuition behind is that the apreciation from training the junior must not be high enough so that the senior devotes her activity exclusively to train the junior. We believe that the training activity must always be performed together with the senior's research work, since she is a scientific researcher in the first place and so places a

\footnotetext{
${ }^{10}$ For the senior scientist, training may increase visibility and reputation when the young professional is a productive member. The model also includes the trainer's inner satisfaction in passing along knowledge.
} 
minimum valuation on the outcome of her own work. ${ }^{11}$ Note also that the weight of the value of the project is normalized to 1 , in such a way that an increase relative importance of the project in the senior's utility is equivalent to a decrease in $\beta$.

The linearity of our model allows us to develop some of our analyses simultaneously for the situation where information is perfect and for the case of full ignorance. We denote by $a$ the expected innate ability of the junior; that is, $a=\hat{a}$ in case of perfect information and $a=E(\tilde{a})$ in case of full ignorance.

Given the parameters $(\alpha, \delta, \beta, t)$ and the ex-ante expectation about the junior scientist's innate ability $a$ with $a \in\{\hat{a}, E(\tilde{a})\}$, the senior scientist chooses the optimal allocation of time between $e_{R}$ and $e_{G}$ that maximizes the ex-ante (expected) value of her utility:

$$
\begin{gathered}
\operatorname{Max}_{e_{R}, e_{G}}\left\{\alpha e_{R}+\delta a e_{G} e_{R}+\beta a e_{G}\right\} \\
\text { s.t. } \quad e_{R}+e_{G}=t, \quad e_{R} \in[0, t], \quad \text { and } \quad e_{G} \in[0, t] .
\end{gathered}
$$

From the solution to this problem we obtain the result that follows.

Lemma 1 Given $(\alpha, \delta, \beta, t)$ and the ex-ante information about the innate ability of the junior scientist $a$, with $a \in\{\hat{a}, E(\tilde{a})\}$, the senior scientist's allocation of time among the tasks of research and training is:

i) When $a \geq \frac{\alpha}{\beta+\delta t}$,

$$
e_{R}^{*}=\frac{t}{2}+\frac{\alpha-\beta a}{2 a \delta} \quad \text { and } \quad e_{G}^{*}=\frac{t}{2}-\frac{\alpha-\beta a}{2 a \delta}
$$

ii) When $a<\frac{\alpha}{\beta+\delta t}$,

$$
e_{R}^{*}=t \quad \text { and } \quad e_{G}^{*}=0
$$

To understand the senior scientist's allocation of time, consider as a reference point the half-half distribution of time $t$. The interior solution depicted in Lemma 1

$$
\left(e_{R}^{*}=\frac{t}{2}+\frac{\alpha-\beta a}{2 a \delta}, e_{G}^{*}=\frac{t}{2}-\frac{\alpha-\beta a}{2 a \delta}\right)
$$

\footnotetext{
${ }^{11}$ One can also argue that the senior researcher has incentives to devote a minimum amount of time to research so as to produce a valuable knowledge. Valuable projects in the past may improve the possibility of financing for future scientific projects. However, for completeness, the case $\beta>\delta t$ is included in the working paper version of the model: Freitas and Macho-Stadler (2011).
} 
shows the deviation from this half-half allocation of time as a function of the senior scientist's effectiveness in the research process $(\alpha)$, the complementarity among the senior's and junior scientist's participation $(\delta)$, the expected innate ability of the junior $(a)$ and the senior's concern about the junior's training $(\beta)$. In the extreme case, the deviation leads to allocate all the time to research. This is the case when the junior has a low expected ability (then he does not receive any training). ${ }^{12}$ The comparative statics go as expected:

Corollary 2 For the combination of parameters satisfying $\alpha \geq a(\beta+\delta t)$ (region $i$ in Lemma 1), more efficiency or more incentives in seniors research, $\alpha$, increase the senior scientist's research effort $e_{R}^{*}$ (and decrease the training effort), while a higher ability of the junior a or a more important participation of the junior in the success of the project $\delta$ increases the training effort $e_{G}^{*}$ (and decreases the research one). Finally, both efforts increase with the time t the senior scientist has to work.

The prediction on the positive effect of the junior's ability $a$ in his training is in accordance with stylized facts. Using a $5 \frac{1}{2}$ year longitudinal investigation over $233 \mathrm{PhD}$ students, Paglis et al. (2006) analyze the effects of supervisory mentoring of advisors on $\mathrm{PhD}$ students in the applied sciences. The results show that supervisory mentoring increases the productivity and the self-efficacy of PhD students. Most importantly, a positive relationship between student potential (ability, experience and commitment to the training) and the extent to which training functions are provided by the faculty advisor is identified. That is, students who show more promise to be productive researchers receive more supervisory training and mentoring from the advisor.

With respect to $\beta$, when $\beta a=\alpha$, the senior scientist will exert an equal effort toward both tasks, $\frac{t}{2}$. For a higher $\beta$, the senior is relatively more concerned about training and the effect on her reputation, so she exerts a greater amount of effort to training in detriment to research. Because the efforts are linear in this parameter, we observe a

\footnotetext{
${ }^{12}$ Given our assumption $\beta \leq \delta t$ we do not have the other extreme case: when the senior scientist's concern about the junior's training is high as compared to the time available that she decides only to perform training. This posibility is taken into consideration in a previous version of our model (Freitas and Macho-Stadler, 2012) where $\beta$ is not constrained from above.
} 
constant rate of substitution. Note that even if $\beta$ goes to 0 , the senior scientist may be interested in allocating time to training if the participation of the junior in the success of the project is important. To make this point clear, let us take the example $\delta=1$. Then the important comparison is in between $\alpha$ and $a$. If the productivity of the senior scientist is high, $\frac{\alpha}{t}>a$, she will just allocate time to research and the project will be run exclusively on her effort. If $\alpha$ is low when compared to $a$ then she will allocate time to training because the complementarity of her research effort with the junior scientist will be the motor of the project. When $\beta$ goes to $\delta t$ then the research effort goes to $\frac{\alpha}{2 a \delta}$ (the minimum time allocated to this task) and training goes to $t-\frac{\alpha}{2 a \delta}$.

Let us now consider two parameters at the time. Imagine that projects or disciplines differ in the characteristics $(\alpha, \delta)$. Since both parameters affect the allocation of effort in different directions, ceteris paribus, we can predict that projects having a high $\alpha$ and a low $\delta$ (resp. a low $\alpha$ and a high $\delta$ ) will induce low (resp. high) training effort. In other words, the model suggests that training tends to be jeopardized in disciplines where the participation of the junior in the success of the seniors own research is not important or the seniors incentives on her own research are strong. For projects where $\alpha$ and $\delta$ are both high or both low it is more difficult to make a prediction since the allocation of effort will be more incline to one or the other activity depending of the forces at work.

Let us consider now situations that differ only in total time available $t$ and the ability of the junior $a$. For a constellation of situations defined by $(a, t)$ both parameters are aligned in their effect on training, but induce different effects on research. When considering two situations where $a$ increases and $t$ decreases, the research effort will certainly be lower but the time devoted to training may decrease or increase. This raises the question of the possible relationship between these two parameters, time available and ability of the junior, that we analyze in Section 4. 


\section{Project Value and Junior Scientist Capability at the Optimal Time Allocation}

Let us now focus on the effect of the optimal time allocation on the project value, $v$, as well as on the junior scientist capability, $q$. We consider first the expected project value and the expected junior scientist capability, i.e., the ex-ante values. Second, we study these variables ex-post.

Ex-ante values Using the results of Lemma 1, we compute the ex-ante (expected) equilibrium levels of $v$ and $q$. We find that, in equilibrium, the ex-ante expected levels of the project and the training are:

i) When $a \geq \frac{\alpha}{\beta+\delta t}$,

$$
v^{*}(a)=\frac{(\alpha+a \delta t)^{2}-(\beta a)^{2}}{4 a \delta} \text { and } q^{*}(a)=\frac{t a}{2}-\frac{\alpha-\beta a}{2 \delta} .
$$

ii) Otherwise,

$$
v^{*}(a)=\alpha t \text { and } q^{*}(a)=0 .
$$

Note that when $a \geq \frac{\alpha}{\beta+\delta t}$ (see Lemma 1) and a strictly positive amount of time is allocated to both tasks, the comparative statics of $v^{*}(a)$ and $q^{*}(a)$ as a function of $\alpha, \beta$, $t$, and $a$ are as expected. ${ }^{13}$ The value of the project increases and the training decreases for higher values of $\alpha$ and lower values of $\beta$ and $a$, and both increase if the senior has more time $t$ available. The effect of $\delta$ is more interesting since it depends on the level of complementarities. The effect of $\delta$ on $v^{*}(a)$ is positive for $a>\frac{\alpha}{\sqrt{\beta^{2}+t^{2} \delta^{2}}}$, and the effect of $\delta$ on $q^{*}(a)$ is positive for $a<\frac{\alpha}{\beta}$. Note also that when $a \in\left(\frac{\alpha}{\sqrt{\beta^{2}+t^{2} \delta^{2}}}, \frac{\alpha}{\beta}\right)$ changes in $\delta$ increase both $v^{*}(a)$ and $q^{*}(a)$.

Ex-post values We now compute the ex-post project value and the ex-post junior scientist's capability that are obtained in equilibrium. Under full information about the innate ability of the junior ex-ante and ex-post values coincide. ${ }^{14}$ We use this case as a

\footnotetext{
${ }^{13}$ The function $v^{*}(a)$ is convex in $a$ and $q^{*}(a)$ is linear in $a$.

${ }^{14}$ With perfect information about the junior scientist's innate ability $\hat{a}=E(\tilde{a})$, and the ex-post and ex-ante values of the project (resp., the junior capability) are identical.
} 
benchmark to measure the difference between ex-ante and ex-post efficiency under ignorance. When the senior scientist decides on the time allocation of efforts under ignorance, the allocation of time depends on her beliefs $a=E(\tilde{a})$. However, the ex-post project value (resp., the junior capability) depends on the true innate ability of the junior scientist $\hat{a}$. Hence, we denote the ex-post levels as $v^{o}(\hat{a}, E(\tilde{a}))$ and $q^{o}(\hat{a}, E(\tilde{a}))$. As a function of the parameters, the ex-post outcomes are:

i) When $a \geq \frac{\alpha}{\beta+\delta t}$,

$$
v^{o}(\hat{a}, E(\tilde{a}))=\alpha x+\hat{a} \delta x y \text { and } q^{o}(\hat{a}, E(\tilde{a}))=\hat{a} y
$$

where $x=e_{R}^{*}(E(\tilde{a}))$ and $y=e_{G}^{*}(E(\tilde{a}))$, both positive. ${ }^{15}$

ii) When $a<\frac{\alpha}{\beta+\delta t}$,

$$
v^{o}(\hat{a}, E(\tilde{a}))=\alpha t \text { and } q^{o}(\hat{a}, E(\tilde{a}))=0
$$

The ex-post value of the project, in the interior case, $v^{o}(\hat{a}, E(\tilde{a}))$ is linear and increasing in $\hat{a}$. To measure the distortion ex-post on the value of the project, we compute $v^{*}(\hat{a})$ and $v^{o}(\hat{a}, E(\tilde{a}))$ and depict the difference. The ex-post value of the project may be higher or lower under ignorance than under full information. Intuitively, under ignorance the value will be higher when expectations on the innate ability of the junior are high and its true level low. Figure 1 represents the comparison for different levels of $E(\tilde{a})$. Under ignorance, the project value is higher than under perfect information for low levels of the true innate ability and the opposite occurs when it is high. ${ }^{16}$

\section{MIA}

It is interesting to analyze how the ex-post project value under ignorance changes with the senior scientist's prior belief about the ability of the junior scientist, $E(\tilde{a})$. Given

\footnotetext{
${ }^{15} x=e_{R}^{*}(E(\tilde{a}))$ and $y=e_{G}^{*}(E(\tilde{a}))$ are the optimal decisions of the senior to perform research and training, respectively, based on the expected capability of the junior.

${ }^{16}$ Note that this comparison is between the decision of the senior scientist with full information and the decision under ignorance, but none of these decisions may be in accordance with the social optimum. The distortion at the bottom that leads senior scientists to train more juniors under ignorance may be good from a social point of view. The distortion at the top may be a serious concern for the society and may push to be interested in guaranteeing that excellent projects and high-potential junior scientists are better identified. We discuss these issues in Section 5.
} 


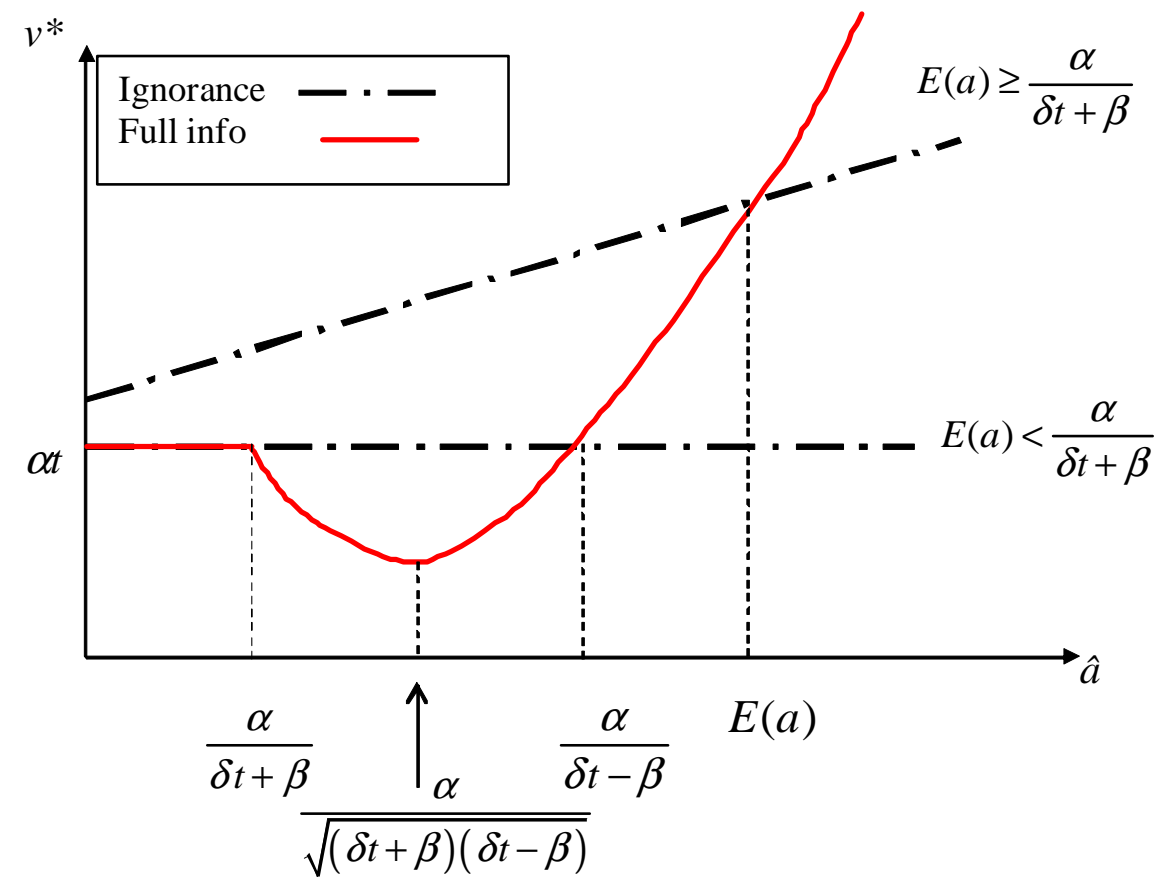

Figure 1: Comparison of the ex-post value of the project under full information $v^{*}(\hat{a})$ and ignorace $v^{o}(\hat{a}, E(a))$. 
$\hat{a}$, when $E(\tilde{a})$ is low enough, ${ }^{17}$ an increase in $E(\tilde{a})$ leads to an increase in $v^{o}(\hat{a}, E(\tilde{a}))$. Otherwise it will decrease. One can also read this result the other way around. Given $E(\tilde{a})$, for high values of $\hat{a}$ a higher prior will increase $v^{o}(\hat{a}, E(\tilde{a}))$, and for low $\hat{a}$ a higher prior will decrease $v^{o}(\hat{a}, E(\tilde{a}))$. This means that a higher prior increases the distortion between $v^{*}(\hat{a})$ and $v^{o}(\hat{a}, E(\tilde{a}))$ for intermediate values of $\hat{a}$ but decreases the distortion for very low and very high values of $\hat{a}$. So, when the junior scientist is indeed very good, this inefficiency becomes smaller as $E(\tilde{a})$ is closer to $\hat{a}$.

We now consider the distortions of the junior scientist's final capability. Note that for the interior solution, $q^{o}(\hat{a}, E(\tilde{a}))$ is linear and increasing in $\hat{a}$ and $E(\tilde{a})$. Comparing the ex-post junior's training under full information $q^{*}(\hat{a})$ and under ignorance $q^{o}(\hat{a}, E(\tilde{a}))$, we obtain the results depicted in Figures 2: there is over-training of low-ability and undertraining of high-ability juniors. The further away the senior scientist's prior is from the true ability of the junior, either from above or below, the higher is the distortion in the ex-post formation, and the distortion increases proportionately. The effect of a higher prior $E(\tilde{a})$ over the junior scientist's ability is a higher slope of the "ignorance" ex-post curve, which means that the distortion increases for lower values of $\hat{a}$ and will decrease for higher values. More accurate training is provided to the population of junior scientists with more potential.

As in the case with the value of the project, the sign of the distortion may be aligned or not aligned with social interest. We will discuss this aspect in Section 5. Note also that if the interval $[\underline{a}, \bar{a}]$ is smaller or if, for a given interval the expected innate ability of the junior scientist, $E(\tilde{a})$ is higher, then it may be the case that more education is provided under ignorance and the distortion is not too big.

\section{Extensions}

Let us consider two natural extensions of our model. In the first one, we allow for the senior scientist to choose the amount of time that she will work. In other words, $t$ is not exogenous but her choice. In the second extension, we assume that the time available

\footnotetext{
${ }^{17}$ More precisely, for $E(\tilde{a}) \leq\left(\frac{\hat{a} \alpha}{2(\alpha+\hat{a} \beta)}\right)^{\frac{1}{2}}$.
} 


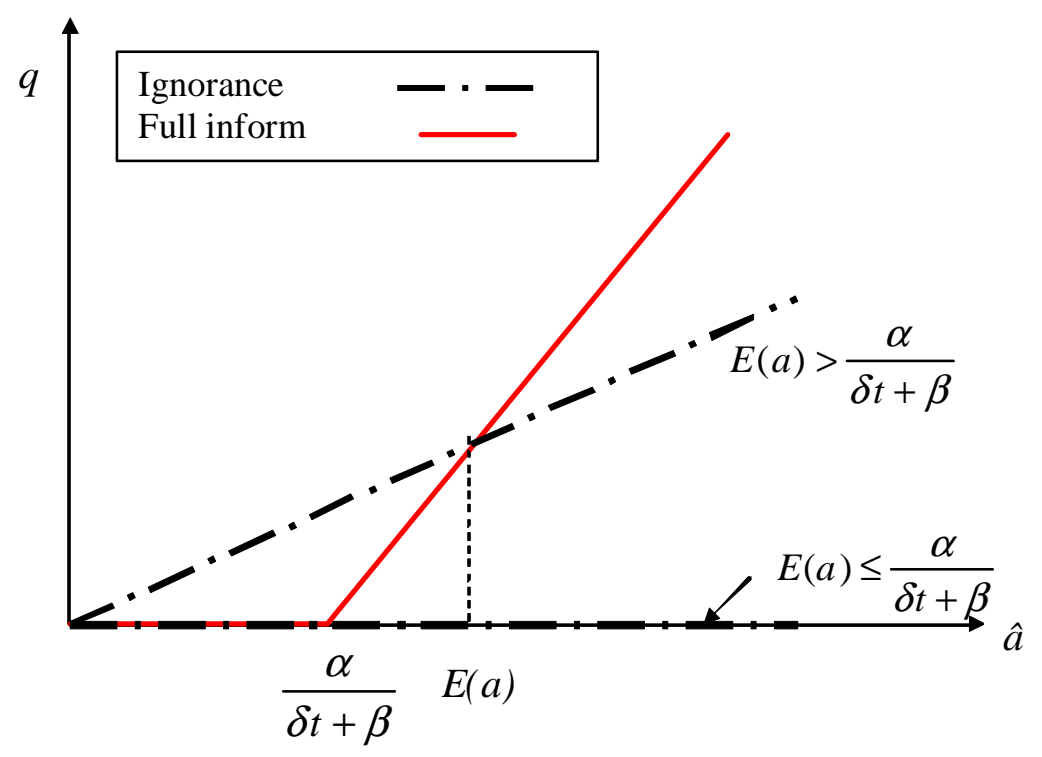

Figure 2: Comparison of the ex-post value of the project under full information $q^{*}(\hat{a})$ and ignorace $q^{o}(\hat{a}, E(a))$. 
is exogenously given, but we allow the senior scientist to have access to a better pool of junior scientists at the cost of some of her time.

Both extensions can be viewed as a sequential decision problem where, once either the time allocated to work or the ability of the junior scientist is determined, the analysis of the previous sections tells us the result in terms of research and training. Hence, it is useful to use the optimal allocation of time as a function of $t$ and $a$ to write the utility of the senior in terms of these variables. Using Lemma 1 we know that:

i) When $a \geq \frac{\alpha}{\beta+\delta t}$,

$$
u^{*}(a, t)=\frac{(a \delta t+\alpha)^{2}-(\beta a)^{2}}{4 a \delta}+\beta\left(\frac{t a}{2}-\frac{\alpha-\beta a}{2 \delta}\right)
$$

ii) When $a<\frac{\alpha}{\beta+\delta t}$,

$$
u^{*}(a)=\alpha t
$$

Total Time Worked The model presented in Section 2 can be understood as a situation where senior scientist motivated to work, and the only decision is how to allocate their time. A complementary situation is to consider that, as in the line of more traditional moral hazard models, the senior scientist may also decide the total amount of time $t$ she will devote to work (the total effort), and she will only work more hours if this is in her interest. In this case, to determine this total working time $t$, the senior scientist would maximize her expected utility net of the cost of the working time, $\frac{c}{2} t^{2}$. The total cost of working is convex in $t$ and it represents the senior's preferences between time spent working and time of leisure. One more unit of time spent working implies giving up one unit of time dedicated to leisure, which is increasingly costly to the senior as she disposes of little amount of time to leisure. We will assume that the cost of working time $c$ is high enough. More precisely, we assume $c \geq \frac{a \delta}{2} \cdot{ }^{18}$ Hence, the senior solves

$$
\underset{t}{\operatorname{Max}}\left\{u^{*}(a, t)-\frac{c}{2} t^{2}\right\}
$$

From this problem, we obtain the following result:

\footnotetext{
${ }^{18}$ If the cost $c$ is smaller that $a \delta / 2$ the optimal time goes to infinite. In this case it would be natural to include a maximum time limit $T$. We will comment on this assumption later, but we will concentrate on the case where $c$ is high for the sake of simplicity.
} 
Lemma 3 The senior scientist's total time as a function of the parameters is

i) When $\alpha-a \beta \geq 0$ and $c \geq \frac{a \delta \alpha}{\alpha-a \beta}$, then

$$
t^{*}=\frac{\alpha}{c}
$$

ii) When $\alpha-a \beta \geq 0$ and $\frac{a \delta}{2}<c \leq \frac{a \delta \alpha}{\alpha-a \beta}$ or $\alpha-a \beta<0$ and $\frac{\beta a^{2} \delta}{a \beta-\alpha}>c$, then

$$
t^{*}=\frac{\alpha+a \beta}{2 c-a \delta}
$$

iii) When $\alpha-a \beta<0$ and $\frac{a \delta}{2}<c \leq \frac{\beta a^{2} \delta}{a \beta-\alpha}$, then

$$
t^{*}=\frac{a \beta}{c}
$$

Note that form Lemma 3 if $\alpha-a \beta \geq 0$, we have the regions depicted in Lemma 3 . The case $\alpha-a \beta<0$ and low $c$ corresponds to the corner solution where time is exclusively allocated to training. From Lemma 3 we conclude that, as expected, the time the senior scientist works $t^{*}$ is a non-decreasing function of $a, \alpha, \beta, \delta$ and is decreasing in $c$. We can interpret $(\alpha, \beta)$ as utility parameters, hence with higher motivation (or incentives) more time the senior will work.

Given the optimal $t^{*}$, we can compute the time allocated to each task. The time allocated to both tasks is decreasing in $c$ (an increase in $c$ now plays a role similar to decrease in $t$ in the previous sections). Effort $e_{R}$ is non-increasing and $e_{G}$ is non-decreasing in the concern for training, $\beta$. More precisely, for $t^{*}=\frac{\alpha}{c}$, the optimal allocation of time is $\left(e_{R}=\frac{\alpha}{c}, e_{G}=0\right)$ and research increases with $\alpha$ but nothing is affected by $\beta$ or $a$. At the other extreme, for $t^{*}=\frac{a \beta}{c}$ the optimal allocation of time is $\left(e_{R}=0, e_{G}=\frac{a \beta}{c}\right)$. For the parameters combinations leading to $t^{*}=\frac{\alpha+a \beta}{2 c-a \delta}$, the time allocated to both tasks deserves some attention. ${ }^{19}$

\footnotetext{
${ }^{19}$ For completeness, let us remark that if $c$ is not constrained from below (i.e., there is a maximum amount of time $T$ that the senior scientist has available), the results will be similar, except for low costs $c\left(c \leq \frac{a \delta}{2}\right)$ and/or $T$ small enough $\left(T<\frac{|\alpha-a \beta|}{a \delta}\right)$. In these cases, the senior chooses to work for all the available time and she allocates all the time $T$ either to research $(\alpha>a \beta)$ or to training $(\alpha<a \beta)$ (except
} 
Corollary 4 When $t^{*}=\frac{\alpha+a \beta}{2 c-a \delta}$ (region ii of Lemma 3), the allocation of time to the tasks is

$$
\begin{aligned}
& e_{R}^{*}=\frac{1}{2}\left(\frac{\alpha+a \beta}{2 c-a \delta}+\frac{\alpha-a \beta}{a \delta}\right) \\
& e_{G}^{*}=\frac{1}{2}\left(\frac{\alpha+a \beta}{2 c-a \delta}-\frac{\alpha-a \beta}{a \delta}\right)
\end{aligned}
$$

The comparative statics of these efforts are presented in Table 1:

\begin{tabular}{|l|l|l|l|l|}
\hline & $\alpha$ & $\beta$ & $a$ & $\delta$ \\
\hline \multirow{2}{*}{$e_{R}^{*}(a, \alpha, \beta, \delta)$} & + & + iff & + iff & + iff \\
& & $c<a \delta$ & $c<\frac{\delta a}{2} \frac{(2 \alpha+a \beta)}{\alpha}$ & $c<\frac{\delta a}{2}\left(\frac{(\alpha+a \beta)^{1 / 2}}{(\alpha-a \beta)^{1 / 2}}-1\right)$ \\
\hline \multirow{2}{*}{$e_{G}^{*}(a, \alpha, \beta, \delta)$} & + iff & + & + & + \\
\hline
\end{tabular}

Table 1

Summarizing, the conclusions of this extension is that the senior will work more hours depending on her motivation. However, results on time allocation are similar to the ones obtained in the Section 2. The difference that when there is a motivation issue, a change in one of the parameters $(a, \alpha, \beta, \delta)$ affects the time allocated to work, and hence corresponds in the basic model to the combine effects of changes in this parameter and $t$. This is the reason why in Corollary 4 both research and training efforts can be increasing with the parameters if the cost of effort $c$ is low enough, or in other words if the time allocated to work suffers an important increase.

When Expected Ability and Time Are Related In Section 2 we analyzed the decisions of a senior scientist that is (randomly) matched with a junior scientist of innate ability $a$. However, one may wonder about what happens if the junior's expected innate ability $a$ depends on some previous activity that the senior performs and that consumes time. This may correspond to a selection process that tries to identify a better population of junior scientists, an advertising or investment procedure that aims to attract a junior if $\alpha=a \beta$, case where she is indifferent). Changes in $\alpha$ or in $\beta$ do not affect the total time allocated to work and only discrete changes may affect to which task this time $T$ is allocated. In these cases, only changes of the time available for these activities may have an effect on the senior scientist's behavior. 
scientist with a higher expected innate ability, or an undergraduate system that provides better skills and better information about the juniors' abilities. Here, we model the relationship between the time invested in increasing the innate ability of the junior she works with and the remaining time available for research and training.

Let us assume that $T$ is the maximum amount of time available for the three tasks and $A$ the expected innate ability of the junior scientist if no effort is select a better junior form the population. Let us denote by $g(T-t)$, with $g>0$, the improvement of the expected innate ability of the junior scientist that the senior can obtain by using an amount of time $(T-t)$ to select (or improve) the quality of the junior with whom she works, in such a way that she will have $t$ to allocate to the tasks of research and formation. Hence, the expected ability of the junior scientist with whom the senior will work with is $a=A+g(T-t)$.

In this case, the senior scientist chooses $(a, t)$ by maximizing her utility function, taking into account the constraints $a=A+g(T-t)$. To simplify presentation and to avoid cumbersome calculations for different regions of parameters, we just present an example where we assume that the senior has no appreciation of the junior scientist's final capability $(\beta=0)$ and that the complementarity effect is $\delta=1$. Under this parameter combination, as a function of $t$, the senior's allocation of time depends on whether $a \geq \frac{\alpha}{\delta t}$ (and her time will be allocated to research and formation) or $a \leq \frac{\alpha}{\delta t}$ (and she will only do research).

Lemma 5 Assuming $\beta=0, \delta=1$ and for a relation between time and ability given by $a=A+g(T-t)$, the senior scientist's decision on the optimal innate ability and on the optimal amount of time spent in previous activities is:

i) When $\frac{(g T+A)^{2}}{12 g} \geq \alpha$,

$$
a^{*}=\frac{g T+A+\sqrt{(g T+A)^{2}-12 \alpha g}}{6} \text { and } t^{*}=\frac{5(g T+A)-\sqrt{(g T+A)^{2}-12 \alpha g}}{6 g}
$$

ii) When $\frac{(g T+A)^{2}}{12 g}<\alpha$,

$$
a^{*}=A \text { and } t^{*}=T
$$


Lemma 5 illustrates that in projects where the productivity of the senior scientist's direct research $(\alpha)$ is low enough, the senior is willing to spend time in selection activities that allow to work with a junior scientist of higher expected innate ability. This is because being a less productive scientist, the senior will want to increase the prospects of working with a more talented junior scientist. When $\alpha$ is high enough, then she will choose not to spend any time in activities to retrieve more information about the junior's innate ability, leaving it at level $A$. This way, she chooses to allocate all of the time resources to training and research only. Note also that for a given combination of the other parameters $(g, \alpha)$, when $T$ or $A$ are small it is more often the case that the senior's optimal decision is not spend time in improving the innate ability of the junior (while this does not mean that she will not allocate some time to formation).

\section{Discussion on Policy Instruments}

We would like to consider here the point of view of a regulator who is concerned both about the level of research that the senior scientist achieves and about the final capacity of the junior as a measure of the potential of the next generation of researchers.

For the shake of the discussion, consider a regulator whose welfare function has the form:

$$
W=E\left(v^{o}(\hat{a}, E(\tilde{a}))\right)+\lambda E\left(q^{o}(\hat{a}, E(\tilde{a}))\right),
$$

where $\lambda>0$ can be interpreted as the society's relative concern about the capability of the next generation of researchers. If $\lambda=0$ the regulator is only concerned by research, while if $\lambda$ goes to infinite, he is only concern by training.

Only when there is no motivation problem (the time $t$ the senior works is fixed) and $\lambda$ coincides with $\beta$, the decision of the senior and the aims of the society concur. If there is a motivation problem or $\lambda$ and $\beta$ do not coincide, the social planner may be tempted to intervene.

An alternative way of looking at the comparative static of the results in Lemma 1 and Corollary 4 is to consider how society may affect the time a senior scientist works and her allocation of time to research and formation. For this purpose, the regulator must affect 
the senior's utility $u=\alpha e_{R}+\delta a e_{G} e_{R}+\beta a e_{G}$, by inducing changes in the parameters $\alpha$, $\delta$ and/or $\beta$ via the instruments available. ${ }^{20}$

If the outcome of research $v$ and the outcome of training $q$ are verifiable and incentives can be based on both of them, the regulator can manipulate the decision of the senior scientist by changing her awareness about these two variables. The regulator can increase the senior scientist's utility from the project's value (increasing $\alpha$ and $\delta$ or, equivalently decreasing $\beta$ ) or he can increase the senior's payoff as a function of the quality of the junior she mentors (increasing $\beta$ ) via the definition of a successful career or the allocation of research funds that weight these aspect of the academic career. Note that, when the total time that the senior works is fixed, in order to change the allocation of time affecting the perceptions of both tasks is useless, while using instruments related to the two tasks at the same time may be quite effective if there is a motivation problem in addition to the allocation one. Also, if for any reason only publications (and other measures of the senior scientist project results) can be used in the scientist's contract, the social planner can encourage more time to research through wages, tenure tack rules, opportunities to travel and access to research funds, or peer esteem. These instruments rewarding research may have a positive or a negative effects on the time allocated to training. If there is no a motivation problem, and these policy are not able to incline the senior researcher to work more hours, then training will suffer. In this case, only by discouraging research can the time allocated to training be increased. In contrast, if motivation is an issue, by rewarding research the social planner may also induce more training. As shown in Corollary 4, if the cost of the effort or the quality of the junior is high enough, then increases in $(\alpha, \delta)$, which correspond to a higher utility associated to the value of the research project, in the same way that increases in $\beta$ induce more research and more training (because they induce more incentives to work). If juniors are gifted enough, both types of instruments (increasing the utility the senior scientist receives from research or from training) have positive effects on the senior scientist's dedication to both tasks. In a society where the population of juniors is of low expected ability, the instruments tend to have positive effects on one task and negative on the other and encouraging one activity crowds out the

\footnotetext{
${ }^{20}$ In some cases the regulator can also change the time available for these tasks $t$ (for example, by reducing the senior's involvement in other time-consuming tasks, such as administrative ones).
} 
effort on the other one. This emphasizes the importance of attracting a good population of junior scientists.

This comment connects with the analysis conducted in Section 4 where Lemma 5 draws attention to the possibility that the population of juniors can be linked to the time allocated to select them. Note however, that measures that increase $a^{*}$ will decreases $t^{*}$. This may lead to an increase in the senior's dedication to a task but may trigger a decrease in the time allocate to the other task unless the cost of obtaining better pools of junior scientists, $g$, decreases. The social planner may want to consider influencing the junior scientist's innate quality $a$, for example, by having an attractive and selective program of fellowships that allows the attraction of better students. Indeed, several European expert institutions (e.g., EURAB, ESF) have given priority to the training of scientists and developed actions so that postdoctoral researchers ascend to PIs in recent years. These actions involve providing access to special grants, as well as promoting free and secure mobility.

\section{Conclusion}

In this paper we provide a model where not only scientists' research results are important but also their involvement in training young scientists. We evaluate the effects of training on he capability of juniors to become successful independent researchers in the future.To this aim we propose a multitask model where the training of a junior scientist depends on the incentives that a senior scientist has to allocate time to training when this is at the cost of spending less time doing research. Our model proposes the (testable) predictions that researchers are more inclined to be involved in training the more time they have to allocate to these tasks, the higher the expected innate ability of the junior scientist, and the less important is her own involvement for the success of the research project is as compared to the complementarities of working with a competent junior.

We study the possibility that a senior scientist engages in previous activities to obtain more information on the innate ability of the junior scientist. This possibility is used when her own productivity on the project is not too high and investing in this activity provides better chances of working with a good junior. We also analyze the distortions 
that arise in the value of a research project and in the final capability of the junior scientist between imperfect information (ex-ante efficiency) and full information (ex-post efficiency). The patterns of the distortions will vary with the relevant parameters, and more or less training may be provided when we compare the decision of the senior under ignorance or full information. This aspect is also important when a regulator considers policies to induce more training and decrease these distortions. We also discuss that, in some cases, if a regulator implements attractive, recognized training programs in earlier education (leading to a higher proportion of good junior scientists), as well as a tougher selection process for research under supervision and attractive conditions to appointments, the proportion and the quality level of independent scientists in the population will be positively affected.

This model is a first approach to the issue. It is to note that we focus on the studentsupervisor relationship, abstracting from the fact that senior scientists can train more junior scientists in the lab. We understand that this would imply a different framework, one that must take into account the different synergies between juniors in the training process. We believe that, in this case, there would be a higher incentive to provide higher training if the lab performs tasks that are very independent between juniors, and lower training when synergies are high since the senior relies more on team training.

We consider that it may help to provide a simple framework and theoretical implications to conduct some empirical studies and to think about policy issues. This model has the potential to be generalized toward the study of additional problems such as the matching of senior and junior scientists, the senior's choice of a research project, or the team component of some research processes. 


\section{APPENDIX}

\subsection{Proof of Lemma 1}

The program can be rewritten as

$$
\begin{gathered}
\underset{e_{R}, e_{G}}{\operatorname{Max}} \alpha e_{R}+a \delta\left(t-e_{R}\right) e_{R}+\beta a\left(t-e_{R}\right) \\
\text { s.t. } \quad e_{R} \geq 0 \quad \text { and } \quad e_{R} \leq t
\end{gathered}
$$

The Lagrangian of this program is $\mathcal{L}=\alpha e_{R}+a \delta\left(t-e_{R}\right) e_{R}+\beta a\left(t-e_{R}\right)+\lambda e_{R}+\mu\left(t-e_{R}\right)$, which FOC is:

$$
\alpha+a \delta\left(t-2 e_{R}\right)-\beta a+\lambda-\mu=0 .
$$

Then cases to consider are

(1) $\lambda=0, \mu=0$ and $e_{R}=\frac{a \delta t+\alpha-\beta a}{2 a \delta}$, which is a candidate when $\frac{a \delta t+\alpha-\beta a}{2 a \delta} \geq 0$ and $\frac{a \delta t+\alpha-\beta a}{2 a \delta} \leq t$, or equivalent, when $\alpha+a(\delta t-\beta) \geq 0$ (which is always the case since $\delta t \geq \beta \geq 0)$ and $\alpha-a(\delta t+\beta) \leq 0$.

(2) $\lambda>0, \mu=0$ and $e_{R}=0$, which is a candidate when $\alpha+a(\delta t-\beta) \leq 0$ and $\alpha-a(\delta t+\beta) \leq 0$. This case never occurs since $\delta t \geq \beta \geq 0$.

(3) $\lambda=0, \mu>0$ and $e_{R}=t$, which asks for $\alpha-a(\delta t+\beta) \geq 0$.

Finally, the combination of these cases gives the results presented in the Lemma.

\subsection{Proof of Lemma 3}

We proceed for simplicity by regions of parameters:

Case 1: $\alpha-a \beta>0$

In this case:

$$
\begin{aligned}
& u(t)=\frac{(a \delta t+\alpha)^{2}-(\beta a)^{2}}{4 a \delta}+\frac{2 \beta a(a \delta t-\alpha+\beta a)}{4 a \delta} \text { if } t \geq \frac{\alpha-a \beta}{a \delta} \\
& u(t)=\alpha t \text { if } t \leq \frac{\alpha-a \beta}{a \delta}
\end{aligned}
$$

Step (i) Let's first consider the function $u(t)=\alpha t$. The maximization problem is

$$
\begin{gathered}
\operatorname{Max}_{t}\left\{\alpha t-\frac{c}{2} t^{2}\right\} \\
\text { s.t. } \quad 0 \leq t \leq \frac{\alpha-a \beta}{a \delta}
\end{gathered}
$$


The Lagrangian function is $\mathcal{L}=\alpha t-\frac{c}{2} t^{2}+\lambda\left(\frac{\alpha-a \beta}{a \delta}-t\right)+\mu t$. The FOC is

$$
\alpha-c t-\lambda+\mu=0 \text {. }
$$

Then the possibilities are (i.1) $\lambda=0$ and $\mu=0$, then $t=\frac{\alpha}{c}$, which is a candidate when $\frac{\alpha}{c} \leq \frac{\alpha-a \beta}{a \delta} \Longleftrightarrow \frac{a \delta \alpha}{\alpha-a \beta} \leq c$. (ii.2) $\lambda>0$ and $\mu=0$, then $t=\frac{\alpha-a \beta}{a \delta}$, which is a candidate when $\alpha-c\left(\frac{\alpha-a \beta}{a \delta}\right)>0$, i.e., $\frac{\alpha}{c}>\frac{\alpha-a \beta}{a \delta} \Longleftrightarrow \frac{a \delta \alpha}{\alpha-a \beta}>c$. (ii.3) $\lambda=0$ and $\mu>0$, then $t=0$, which is a candidate if $\alpha<0$ which is never the case.

Step (ii) Let's now consider the function $u(t)=\frac{(\alpha+a t \delta)^{2}-(a \beta)^{2}}{4 a \delta}+\frac{2 a \beta(a \delta t-\alpha+a \beta)}{4 a \delta}$. The maximization problem is

$$
\begin{gathered}
\operatorname{Max}_{t}\left\{\frac{(\alpha+a t \delta)^{2}-(a \beta)^{2}}{4 a \delta}+\frac{2 a \beta(a \delta t-\alpha+a \beta)}{4 a \delta}-\frac{c}{2} t^{2}\right\} \\
\text { s.t. } \quad t \geq \frac{\alpha-a \beta}{a \delta}
\end{gathered}
$$

The Lagrangian is $\mathcal{L}=\frac{(\alpha+a t \delta)^{2}-(a \beta)^{2}}{4 a \delta}+\frac{2 a \beta(a \delta t-\alpha+a \beta)}{4 a \delta}-\frac{c}{2} t^{2}+\lambda\left(t-\frac{\alpha-a \beta}{a \delta}\right)$. The FOC is

$$
\frac{\alpha+a t \delta+a \beta}{2}-c t+\lambda=0 .
$$

Then, the possibilities are: (ii.1) $\lambda=0$ and $t=\frac{\alpha+a \beta}{2 c-a \delta}$, which is a candidate when $\frac{\alpha+a \beta}{2 c-a \delta}>$ $\frac{\alpha-a \beta}{a \delta}$ or, equivalently, when $\frac{a \delta \alpha}{\alpha-a \beta} \geq c$. (ii.2) $\lambda>0$ and $t=\frac{\alpha-a \beta}{a \delta}$, which asks for $\frac{\alpha+a \delta\left(\frac{\alpha-a \beta}{a \delta}\right)}{2}-$ $c\left(\frac{\alpha-a \beta}{a \delta}\right)<0$, or equivalently $\frac{a \delta \alpha}{\alpha-a \beta}<c$.

Step (iii) Comparing the results from the previews steps, and realizing that the candidate for solution in one of cases is a possible solution in the other case we obtain the result presented in the Lemma.

Case 2: $\alpha-a \beta<0$

Here we have:

$$
\begin{aligned}
& u(t)=\frac{(a \delta t+\alpha)^{2}-(\beta a)^{2}}{4 a \delta}+\frac{2 \beta a(a \delta t-\alpha+\beta a)}{4 a \delta} \text { if } t \geq \frac{a \beta-\alpha}{a \delta} \\
& u(t)=a \beta t \text { if } t \leq \frac{a \beta-\alpha}{a \delta}
\end{aligned}
$$

Step (i) Let's consider the function $u(t)=a \beta t$. The maximization problem is

$$
\begin{gathered}
\underset{e_{R}, e_{G}}{\operatorname{Max}}\left\{a \beta t-\frac{c}{2} t^{2}\right\} \\
\text { s.t. } \quad 0 \leq t \leq \frac{a \beta-\alpha}{a \delta}
\end{gathered}
$$


The Lagrangian function is $\mathcal{L}=a \beta t-\frac{c}{2} t^{2}+\lambda\left(\frac{a \beta-\alpha}{a \delta}-t\right)+\mu t$. The FOC is

$$
a \beta-c t-\lambda+\mu=0 .
$$

Then the possibilities are (i.1) $\lambda=0$ and $\mu=0$ which gives $t=\frac{a \beta}{c}$, which is a candidate when $\frac{a \beta}{c} \leq \frac{a \beta-\alpha}{a \delta} \Longleftrightarrow \frac{\beta a^{2} \delta}{a \beta-\alpha} \leq c$. (ii.2) $\lambda>0$ and $\mu=0$, and $t=\frac{a \beta-\alpha}{a \delta}$, which is a candidate when $\alpha-c\left(\frac{a \beta-\alpha}{a \delta}\right)>0$, i.e., $\frac{\alpha}{c}>\frac{a \beta-\alpha}{a \delta} \Longleftrightarrow \frac{\beta a^{2} \delta}{a \beta-\alpha}>c$. (ii.2) $\lambda=0$ and $\mu>0$, and $t=0$, which is a candidate when $a \beta<0$, which is never the case.

Step (ii) Let's now consider the function $u(t)=\frac{(\alpha+a t \delta)^{2}-(a \beta)^{2}}{4 a \delta}+\frac{2 a \beta(a \delta t-\alpha+a \beta)}{4 a \delta}$. The maximization problem is

$$
\begin{gathered}
\operatorname{Max}_{t}\left\{\frac{(\alpha+a t \delta)^{2}-(a \beta)^{2}}{4 a \delta}+\frac{2 a \beta(a \delta t-\alpha+a \beta)}{4 a \delta}-\frac{c}{2} t^{2}\right\} \\
\text { s.t. } \quad t \geq \frac{a \beta-\alpha}{a \delta}
\end{gathered}
$$

The Lagrangian function is $\mathcal{L}=\frac{(\alpha+a t \delta)^{2}-(a \beta)^{2}}{4 a \delta}+\frac{2 a \beta(a \delta t-\alpha+a \beta)}{4 a \delta}-\frac{c}{2} t^{2}+\lambda\left(t-\frac{a \beta-\alpha}{a \delta}\right)$. The FOC is

$$
\frac{\alpha+a t \delta+a \beta}{2}-c t+\lambda=0 .
$$

Then, the possibilities are: (ii.1) $\lambda=0$ and $t=\frac{\alpha+a \beta}{2 c-a \delta}$, which is a candidate when $\frac{\alpha+a \beta}{2 c-a \delta}>$ $\frac{a \beta-\alpha}{a \delta}$ or, equivalently, when $\frac{\beta a^{2} \delta}{a \beta-\alpha}>c$. Note that $\frac{\beta a^{2} \delta}{a \beta-\alpha}<\frac{a \delta}{2}$ (ii.2) $\lambda>0$ and $t=\frac{a \beta-\alpha}{a \delta}$, which asks for $\frac{\alpha+a \delta\left(-\frac{\alpha-a \beta}{a \delta}\right)+a \beta}{2}-c\left(\frac{a \beta-\alpha}{a \delta}\right)<0$, or equivalently $\frac{\beta a^{2} \delta}{a \beta-\alpha}<c$.

Step (iii). Comparing the results from the previews steps, and realizing that the candidate for solution in one of cases is a possible solution in the other case we obtain the result presented in the Lemma.

Case 3: $\alpha-a \beta=0$

In this case:

$$
u(t)=t \frac{2(\alpha+\beta a)+a \delta t}{4} \text { if } t \geq 0
$$

Then the maximization problem of the senior is:

$$
\begin{gathered}
\underset{t}{\operatorname{Max}}\left\{\frac{2 t(\alpha+\beta a)+a \delta t^{2}}{4}-\frac{c}{2} t^{2}\right\} \\
\text { s.t. } \quad t \geq 0
\end{gathered}
$$


The Lagrangian function is $\mathcal{L}=\frac{2 t(\alpha+\beta a)+a \delta t^{2}}{4}-\frac{c}{2} t^{2}+\lambda t$. The FOC is

$$
\frac{\alpha+a \beta+a t \delta}{2}-c t+\lambda=0
$$

Then, the possibilities are: (i) $\lambda=0$ and $t=\frac{\alpha+a \beta}{2 c-a \delta}$, which is a candidate when $\frac{\alpha+a \beta}{2 c-a \delta} \geq 0$ or, equivalently, when $c>\frac{a \delta}{2}$. (ii) $\lambda>0$ and $t=0$, which asks for $\frac{\alpha+a \beta}{2}<0$, which is never the case.

\subsection{Proof of Lemma 5}

For the case $\beta=0$ and $\delta=1$, we consider the candidates that satisfy $a^{2}-(g T+A) a+\alpha g \geq$ 0 and $a^{2}-(g T+A) a+\alpha g<0$ sequentially and then we provide the solution as function of the parameters.

Step 1: $a^{2}-(g T+A) a+\alpha g \geq 0$

The utility function to be considered is $u^{*}(t)=\alpha\left(T-\frac{a-A}{g}\right)$.

Depending on the parameters, the values of $a$ such that $a^{2}-(g T+A) a+\alpha g=0$ (they only exist if $\left.(g T+A)^{2}-4 \alpha g \geq 0\right)$ will lie, or not, in the interval where the junior's ability is defined, $[A, g T+A]$. When $a=A, a^{2}-(g T+A) a+\alpha g$ has a positive value if $A T \leq \alpha$, and a negative value otherwise. When $a=g T+A$, the value for $a^{2}-(g T+A) a+\alpha g$ is always positive. Hence we can define 3 possible regions to attain a solution: a) when $A T \leq \alpha$ and $(g T+A)^{2}-4 \alpha g \geq 0 ;$ b) when $A T \leq \alpha$ and $(g T+A)^{2}-4 \alpha g<0$; and c) when $A T>\alpha$ and $(g T+A)^{2}-4 \alpha g \geq 0$. The One last case could be $A T>\alpha$ and $(g T+A)^{2}-4 \alpha g<0$, however it is not possible since this would mean that the function never has negative values and, simultaneously, has a negative value when $a=A$.

Case a) $A T \leq \alpha$ and $(g T+A)^{2}-4 \alpha g \geq 0$

In this case the optimal ability lies in either one of the two following regions: $A \leq a \leq$ $\frac{g T+A-\sqrt{(g T+A)^{2}-4 \alpha g}}{2}$ or $\frac{g T+A+\sqrt[2]{(g T+A)^{2}-4 \alpha g}}{2} \leq a \leq g T+A$. We first formalize the problem with respect to the first region:

$$
\begin{aligned}
& \operatorname{Max}_{a}\left\{\alpha\left(T-\frac{a-A}{g}\right)\right\} \\
\text { s.t } \quad a \geq A & \\
a & \leq \frac{g T+A-\sqrt{(g T+A)^{2}-4 \alpha g}}{2}
\end{aligned}
$$


The lagrangian is $\mathcal{L}=\alpha\left(T-\frac{a-A}{g}\right)+\lambda(a-A)+\mu\left(\frac{g T+A-\sqrt{(g T+A)^{2}-4 \alpha g}}{2}-a\right)$. The FOC is $-\frac{\alpha}{g}+\lambda-\mu=0$

There are two possible cases for the lagrangian multipliers:

1) $\lambda>0, \mu=0 . a=A$ is a candidate.

2) $\lambda>0, \mu>0$. This holds when $A=\frac{g T+A-\sqrt{(g T+A)^{2}-4 \alpha g}}{2} \Leftrightarrow A T=\alpha$ which is a particular case of 1 ). Hence $a=A$ is again a candidate.

Formalizing the problem with respect to the second region, the FOC is:

$$
-\frac{\alpha}{g}-\lambda+\mu=0
$$

One possible case exists for the lagrange multiplier:

1) $\lambda=0, \mu>0$. In this case, $a=\frac{g T+A+\sqrt{(g T+A)^{2}-4 \alpha g}}{2}$ is a candidate.

Since the utility function is decreasing in $a, a=A$ is a candidate for the optimal ability.

Case b) $A T \leq \alpha$ and $(g T+A)^{2}-4 \alpha g<0$

The region to work with is $A \leq a \leq g T+A$, since the function always has positive values in this case. Since the utility function of the senior is decreasing in $a, a=A$ is a candidate for the optimal ability.

Case c) $A T>\alpha$ and $(g T+A)^{2}-4 \alpha g \geq 0$

This is a particular case of case 1.a), where we only consider the second region, hence $a=\frac{g T+A+\sqrt{(g T+A)^{2}-4 \alpha g}}{2}$ is a candidate for the optimal ability.

We now summarize the candidates for step 1:

$$
\begin{aligned}
& a=A \quad \text { if } \quad A T \leq \alpha \\
& a=\frac{g T+A+\sqrt{(g T+A)^{2}-4 \alpha g}}{2} \quad \text { if } \quad A T>\alpha \text { and }(g T+A)^{2}-4 \alpha g \geq 0
\end{aligned}
$$

Step 2: $a^{2}-(g T+A) a+\alpha g \leq 0$

The utility function to be considered in this case is $u^{*}(a, t)=\frac{(a t+\alpha)^{2}}{4 a}$.

Following the same logic as in step 1, we have 2 subcases to solve this problem: 2.a) when $A T \geq \alpha$ and $\left.(g T+A)^{2}-4 \alpha g \geq 0 ; 2 . b\right)$ when $A T \leq \alpha$ and $(g T+A)^{2}-4 \alpha g \geq 0$. The other two subcases are impossible, since $(g T+A)^{2}-4 \alpha g<0$ means the function always has positive values. Hence, in this step we take as given that $(g T+A)^{2}-4 \alpha g \geq 0$. 
Case a) $A T \geq \alpha$

There is one region for $a$ to work with: $A \leq a \leq \frac{g T+A+\sqrt[2]{(g T+A)^{2}-4 \alpha g}}{2}$. The maximization problem is:

$$
\begin{array}{r}
\operatorname{Max}_{a, t}\left\{\frac{1}{4 a}\left(a\left(T-\frac{a-A}{g}\right)+\alpha\right)^{2}\right\} \\
\text { s.t. } a \geq A \text { and } \quad a \leq \frac{g T+A+\sqrt{(g T+A)^{2}-4 \alpha g}}{2}
\end{array}
$$

The FOC is:

$$
\frac{1}{a^{2}}\left(a\left(T-\frac{a-A}{g}\right)+\alpha\right)\left(a\left(T-\frac{3 a-A}{g}\right)-\alpha\right)+\lambda-\mu=0
$$

There are 4 possible cases for the lagrange multipliers:

1) $\lambda>0, \mu=0$. Looking at the FOC, it must be that $a\left(T-\frac{3 a-A}{g}\right)-\alpha<0$, that is, $a \in\left(\frac{g T+A-\sqrt{(g T+A)^{2}-12 \alpha g}}{6}, \frac{g T+A+\sqrt{(g T+A)^{2}-12 \alpha g}}{6}\right)$, which is the case when $a=A$.

2) $\lambda=0, \mu>0$. In this case, $a=\frac{g T+A+\sqrt[2]{(g T+A)^{2}-4 \alpha g}}{2}$ is a candidate if

$a \in\left(\frac{g T+A-\sqrt{(g T+A)^{2}-12 \alpha g}}{6}, \frac{g T+A+\sqrt[2]{(g T+A)^{2}-12 \alpha g}}{6}\right)$. We check that indeed $a$ belongs to this interval. This holds only if $(g T+A)^{2}-12 \alpha g \geq 0$.

3) $\lambda=0, \mu=0 . a=\frac{g T+A \pm \sqrt[2]{(g T+A)^{2}-12 \alpha g}}{6}$ are candidates, provided $(g T+A)^{2}-12 \alpha g \geq$ 0 .

4) $\lambda>0, \mu>0$. This holds when $A=\frac{g T+A-\sqrt{(g T+A)^{2}-4 \alpha g}}{2} \Leftrightarrow A T=\alpha$. Hence $a=A$ is a candidate again.

The utility function is decreasing from $A$ until $\frac{g T+A-\sqrt{(g T+A)^{2}-12 \alpha g}}{6}$, increasing from then on until $\frac{g T+A+\sqrt{(g T+A)^{2}-12 \alpha g}}{6}$, and decreasing onwards. Hence, when $(g T+A)^{2}-$ $12 \alpha g \geq 0 \quad a=\frac{g T+A+\sqrt{(g T+A)^{2}-12 \alpha g}}{6}$ is candidate for the optimal ability and when $(g T+$ $A)^{2}-12 \alpha g<0, a=A$.

Case b) $A T \leq \alpha$

There is one region for $a$ to work with: $\frac{g T+A-\sqrt{(g T+A)^{2}-4 \alpha g}}{2} \leq a \leq \frac{g T+A+\sqrt{(g T+A)^{2}-4 \alpha g}}{2}$. The FOC is the same as in case a), hence the possible cases for the lagrangean multipliers are:

1) $\lambda>0, \mu=0 . a=\frac{g T+A-\sqrt{(g T+A)^{2}-4 \alpha g}}{2}$ and it must be that $a \in\left(\frac{g T+A-\sqrt{(g T+A)^{2}-12 \alpha g}}{6}\right.$, $\left.\frac{g T+A+\sqrt{(g T+A)^{2}-12 \alpha g}}{6}\right)$. Since $\frac{g T+A-\sqrt{(g T+A)^{2}-4 \alpha g}}{2}<\frac{g T+A-\sqrt{(g T+A)^{2}-12 \alpha g}}{6}$, indeed it is a candidate. 
2) $\lambda=0, \mu>0 . a=\frac{g T+A+\sqrt{(g T+A)^{2}-4 \alpha g}}{2}$ and it must be that $a \in\left(\frac{g T+A-\sqrt{(g T+A)^{2}-12 \alpha g}}{6}\right.$, $\left.\frac{g T+A+\sqrt{(g T+A)^{2}-12 \alpha g}}{6}\right)$. It is straightforward to check that $a$ indeed belongs to this interval, so it is a candidate, provided that $(g T+A)^{2}-12 \alpha g \geq 0$.

3) $\lambda=0, \mu=0$. In this case, $a=\frac{g T+A \pm \sqrt{(g T+A)^{2}-12 \alpha g}}{6}$ provided that $(g T+A)^{2}-12 \alpha g \geq$ 0 .

4) $\lambda>0, \mu>0$. In this case, $a=\frac{g T+A}{6}$, which happens when $(g T+A)^{2}-12 \alpha g=0$, a particular case of 3$)$.

Analyzing all the candidates and the behavior of the utility function, $a=\frac{g T+A+\sqrt{(g T+A)^{2}-12 \alpha g}}{6}$ is a candidate for the optimal ability when $(g T+A)^{2}-12 \alpha g \geq 0$ and $a=\frac{g T+A-\sqrt{(g T+A)^{2}-4 \alpha g}}{2}$ when $(g T+A)^{2}-12 \alpha g<0$.

We now summarize all candidates for case 2 :

$$
\begin{aligned}
& a=\frac{g T+A+\sqrt{(g T+A)^{2}-12 \alpha g}}{6} \quad \text { if } \quad(g T+A)^{2}-12 \alpha g \geq 0 \\
& a=A \quad \text { if } \quad A T \geq \alpha \text { and }(g T+A)^{2}-12 \alpha g<0 \\
& a=\frac{g T+A-\sqrt{(g T+A)^{2}-4 \alpha g}}{2} \quad \text { if } \quad A T<\alpha \text { and }(g T+A)^{2}-12 \alpha g<0
\end{aligned}
$$

As function of the parameters, we evaluate and compare the utility of the solutions attained in step 1 and step 2. The final solutions for $a^{*}$ and $t^{*}$ (attained recursively) are:

- If $(g T+A)^{2}-12 \alpha g \geq 0$, then $a^{*}=\frac{g T+A+\sqrt{(g T+A)^{2}-12 \alpha g}}{6}$ and $t^{*}=\frac{5(g T+A)-\sqrt{(g T+A)^{2}-12 \alpha g}}{6 g}$

- If $(g T+A)^{2}-12 \alpha g<0$, then $a^{*}=A$ and $t^{*}=T$.

\section{References}

[1] Banal-Estañol, A. and Macho-Stadler, I. (2010): "Scientific and Commercial Incentives in R\&D: Research vs. Development", Journal of Economics and Management Strategy 19(1), 185-221.

[2] Cech, T. and Bond, E. (2004): "Managing Your Own Lab", Science June 18, Vol. 304, No. 5678, 1717. 
[3] European Commission (2004): "Increasing Human Resources for Science and Technology in Europe", Report chaired by J.M. Gago, High Level Group (HLG) Human Resources for Science and Technology in Europe.

[4] Frame, I. and Allen, L. (2002): "A Flexible Approach to PhD Research Training", Quality Assurance in Education 12(2), 98-103.

[5] Freitas, A. and Macho-Stadler, I. (2011): "On the Joint Production of Research and Training", Working Paper 531, Barcelona GSE.

[6] Golde, C. (2000): "Should I stay or should I go? Student descriptions of the doctoral attrition process", The Review of Higher Education 23(2), 199-227.

[7] HESA, 2011, "Patterns and Trends Report 2011 in UK Higher Education", http://www.universitiesuk.ac.uk/Publications/Documents/

PatternsAndTrendsinUKHigherEducation.pdf.

[8] Lacetera, N. and Zirulia, L. (2008): "Knowledge Spillovers, Competition, and Taste for Science in a Model of R\&D Incentive Provision", Universita' di Bologna, Working Paper..

[9] Lovitts, B. E. (2001): "Leaving the ivory tower: the causes and consequences of departure from doctoral study", Lanham, MA, Rowman \& Littlefield.

[10] Lovitts, B. E. (2005): "Being a good course-taker is not enough: a Theoretical Perspective on the Transition to Independent Research", Studies in Higher Education $30(2), 137-154$.

[11] Nerad, M. and Cerny, J. (1999): "Postdoctoral Patterns, Career Advancement, and Problems", Science September 3, Vol. 285. No. 5433, 1533-1535.

[12] Paglis, L., Green, S. and Bauer T. (2006): "Does Advisor Mentoring Add Value? A Longitudinal Study of Mentoring and Doctoral Student Outcomes", Research in Higher Education 47(5), 451-476. 
[13] Pole, C., Sprokkereef, A., Burgess, Robert G., and Lakin, E. (1997): "Supervision of Doctoral Students in the Natural Sciences: expectations and experiences", Assessment $\&$ Evaluation in Higher Education 22(1), 49-63.

[14] Puljak, L. (2006): "Career Blocker: Bad Advisors" in http://sciencecareers.sciencemag.org.

[15] Stephan, P. E. and S.G. Levin, 1992, Striking the Mother Lode in Science: The Importance of Age, Place, and Time, New York: Oxford University Press.

[16] Vogel, G. (1999): "A Day in the Life of a Topflight Lab", Science September 3, Vol. 285, 1531-1532.

[17] Walckiers, A. (2008): "Multidimensional Contracts with Task-Specific Productivities: An Application to Universities", International Tax and Public Finance 15, 165-198. 